\title{
Repetition Blindness for Faces: A Comparison of Face Identity, Expression, and Gender Judgments
}

\author{
Karen Murphy ${ }^{1,2}$ and Zoe Ward ${ }^{2}$ \\ ${ }^{1}$ Menzies Health Institute Queensland, Griffith University, Gold Coast, Queensland, Australia \\ ${ }^{2}$ School of Applied Psychology, Gold Coast Campus, Griffith University, Queensland, Australia
}

ABSTRACT

\begin{abstract}
Repetition blindness (RB) refers to the impairment in reporting two identical targets within a rapid serial visual presentation stream. While numerous studies have demonstrated RB for words and picture of objects, very few studies have examined RB for faces. This study extended this research by examining RB when the two faces were complete repeats (same emotion and identity), identity repeats (same individual, different emotion), and emotion repeats (different individual, same emotion) for identity, gender, and expression judgment tasks. Complete RB and identity RB effects were evident for all three judgment tasks. Emotion RB was only evident for the expression and gender judgments. Complete RB effects were larger than emotion or identity RB effects across all judgment tasks. For the expression judgments, there was more emotion than identity RB. The identity RB effect was larger than the emotion RB effect for the gender judgments. Cross task comparisons revealed larger complete RB effects for the expression and gender judgments than the identity decisions. There was a larger emotion RB effect for the expression than gender judgments and the identity RB effect was larger for the gender than for the identity and expression judgments. These results indicate that while faces are subject to $\mathrm{RB}$, this is affected by the type of repeated information and relevance of the facial characteristic to the judgment decision. This study provides further support for the operation of separate processing mechanisms for face gender, emotion, and identity information within models of face recognition.
\end{abstract}


An extensive body of research shows that RB occurs for words and objects (e.g., Arnell \& Jolicoeur, 1997; Bavelier, 1994; Coltheart et al., 2005; Harris \& Dux, 2005; Kanwisher, 1987; Kanwisher \& Yin, 1993; Kanwisher, Yin, \& Wojciulik, 1999). However, very few studies have examined RB for faces (Buttle, 2010; Coltheart, Bornhofen, Mondy, \& Stephenson, 2004; Mondy, Coltheart, \& Stephenson, 2004; Mowszowski, McDonald, Wang, \& Bornhofen, 2012). Coltheart and colleagues (Coltheart et al., 2004; Mondy et al., 2004) demonstrated that RB occurred for famous faces, unfamiliar faces, nonfamous faces with differing facial expressions, and for different photographs of the same person. Buttle (2010) investigated RB for facial expression and gender judgments using four types of stimulus repetition: complete repeat (same individual and emotion), emotion repeat (different individual, same emotion), identity repeat (same individual, different emotion) and no repeat (different individual, different emotion). Emotion repetition did not produce $\mathrm{RB}$ for either the expression or gender judgments. For both judgment tasks, there was RB when $\mathrm{C} 1$ and $\mathrm{C} 2$ were completely identical (repeated identity and emotion) and the same individual with different emotional expressions (repeated identity only), although RB was larger for the complete repeat condition. While these results might suggest that emotion is immune to $\mathrm{RB}$ and that sufficient attentional resources must be available to process emotional information (Holmes, Vuilleumier, \& Eimer, 2003; Pessoa, McKenna, Gutierrez, \& Ungerleider, 2002), they are at odds with research demonstrating that the processing of emotional information is automatic (e.g., Batty \& Taylor, 2003; Mogg \& Bradley, 1999a, 1999b). A number of methodological issues may have impacted on the results of Buttle's study, thereby eliminating emotion RB. The current study will address these issues to further examine the occurrence of RB for faces.

In relation to Buttle (2010), one issue was that the study used the same number of single and dual target trials. This differs from many other RB studies that have used a small number of single target trials (e.g., approximately 5\%) to control for response bias (e.g., Coltheart et al., 2005; Harris \& Dux, 2005). The 50\% proportion of single trials may have biased participants to report only one target in the repeat trials, thereby magnifying RB for the identity and complete repeat conditions. ${ }^{1}$ Another issue was that Buttle only used four faces, which may have increased participants' familiarity with them and improved accuracy overall. ${ }^{2}$ Further, the faces also had visible hairstyles, which might have allowed the gender judgments to be made based on distinguishing features (e.g., hairstyles) rather than via the face recognition mechanisms. Hence, it is possible that the gender judgments were made through feature processing occurring outside the face processing system, whilst the emotion judgments utilized face recognition processes. This study addressed these issues by reducing the number of single target trials, increasing the number of face stimuli, and removing the hairstyles from the face photographs. The aim of the study was to examine the role of different repeated facial features (complete, identity, emotion) in the production of RB for gender, expression, and identity judgments to assess the occurrence of RB for these different processing mechanisms within the face recognition system.
Various models suggest that identity, gender, and expression information are processed by different cognitive and neural mechanisms within the face processing system (e.g., Bruce \& Young, 1986; Duchaine \& Yovel, 2015; Haxby, Hoffman, \& Gobbini, 2000). According to Bruce and Young's (1986) cognitive model of face recognition, initially, faces are processed via a view-centered representation of the face and information about expression and lip movement are processed via separate mechanisms. If the face is familiar, it will activate the face recognition units (memory for the structure of faces-structural codes), while unfamiliar faces are processed via the directed visual processing pathway using pictorial codes of the face representation (Bruce \& Young, 1986; Duchaine \& Yovel, 2015). Thus, facial expression and face identity are processed via interrelated but separate pathways and this separation occurs early on in the visual perceptual processing stages (Fisher, Towler, \& Eimer, 2016).

Neural models of face processing also propose that face characteristics are processed via different pathways within the human brain (e.g., Duchaine \& Yovel, 2015; Haxby et al., 2000). According to these approaches, face invariant aspects such as age, identity, and gender are processed via the ventral visual stream within the brain, which includes the regions of the fusiform face area and the inferior occipital cortex. Imaging data indicates that both familiar and unfamiliar faces are processed within the fusiform face area (e.g., Andrews, DaviesThompson, Kingstone, \& Young, 2010; Gobbini \& Haxby, 2007). Variable facial attributes, such as speech and expression, are processed via a pathway from the occipital cortex to the superior temporal sulcus. Recent evidence suggests that the fusiform face area may also process facial expression (Bernstein \& Yovel, 2015). However, Duchaine and Yovel (2015) suggest that expression processing within the fusiform face area reflects a general sensitivity to shape information, while the face processing area in the superior temporal sulcus responds to faces showing emotion information.

The operation of separate pathways for expression and face identity processing is supported by studies of patients with prosopagnosia, who have impaired face recognition but are able to accurately judge the emotional expressions on faces (e.g., Humphreys, Donnelly, \& Riddoch, 1993). Facial expression judgments activate brain regions (superior temporal sulcus and limbic structures) beyond those associated with face identification (beyond the fusiform face region, e.g., Habel et al., 2007; Hasselmo, Rolls, \& Baylis, 1989; Haxby et al., 2000; Narumoto et al., 2000; Todorov \& Engell, 2008), and fMRI studies show different neural correlates for facial identity and expression decisions (Jehna et al., 2011). Brain damage can produce impairments in emotion processing, leaving face identification unaffected. For example, bilateral amygdala lesions impair fear recognition (Adolphs, 1999; Adolphs, Tranel, Damasio, \& Damasio, 1994; Jehna et al., 2011), and disgust recognition is impaired with disturbed connections of the striatum and basal ganglia (Hennenlotter et al., 2004; Jacobs, Shuren, Bowers, \& Heilman, 1995; Sprengelmeyer et al., 1996). In these cases, face recognition or identification skills remain intact and only emotion processing is impaired. Thus, both the neural and behavioral data 
are consistent with the independent processing of emotion and face identity information within the face recognition system.

It has been suggested that face, gender, and identity information might be processed separately (e.g., Ellis, Young, \& Flude, 1990; Hole \& Bourne, 2010). Some studies have shown that gender decisions are not necessary for face identification, supporting the independent operation of these processes (Calder et al., 2000; Ellis, Flude, Young, \& Burton, 1996; Ellis et al., 1990; Wild et al., 2000; see Zhao \& Hayward, 2010, for a different perspective). Other studies have shown that when participants categorized faces by gender, accuracy was greater than when they were asked to recognize faces from an entire set of faces (Wild et al., 2000) and that gender decisions can occur more rapidly than face identity judgments (Bruyer, Galvez, \& Prairial, 1993). These results support the idea that gender decisions require the discrimination between relevant gender specific attributes of a face, while identification uses configural information (Wild et al., 2000).

Neuropsychological and laboratory studies indicate that gender and identity judgments involve separate brain regions (Bruyer et al., 1993; Mattson, Levin, \& Grafman, 2000; Sergent, Ohta, \& MacDonald, 1992). For example, prosopagnosia patients can report the gender of a face, although they cannot identify the person (Bruyer et al., 1993; Tranel, Damasio, \& Damasio, 1988). An ERP study found that both age and gender categorization tasks activated the fronto-central areas but not the occipito-temporal facial recognition areas (MouchetantRostaing, Giard, Bentin, Aguera, \& Pernier, 2000).

Thus, the empirical evidence supports the operation of different pathways for the gender, expression, and face identity information within the face processing system. To date, no study has investigated these three aspects of face processing in relation to RB. Thus, this study compared $\mathrm{RB}$ for three different face judgment tasks when different components of the faces were repeated (emotion, identity, identity and emotion together). Utilizing three different face judgment tasks would manipulate the attentional demands of each task and allow examination of the impact of this factor on $\mathrm{RB}$ for different repeated face characteristics. By varying the repeated aspect of the face (identity, emotion, or emotion and identity) this study examined the vulnerability of the various face processing pathways to $\mathrm{RB}$. This will determine if overall face identity or only certain facial features (emotion, gender) are susceptible to $\mathrm{RB}$.

\section{METHOD}

\section{Participants}

Thirty-one (24 females, seven males) first year psychology students, aged between 17 and 65 years $\left(M_{\text {age }}=23.42, S D=10.87\right)$ completed this study in return for course credit. All participants had normal or corrected to normal vision. The study was approved by the University Human Ethics Committee and participants provided written consent before completing the experiment. The data of five participants were excluded from the analysis due to poor performance on the single target catch trials in at least one judgment task (less than 70\% accuracy, typically repeat responses). This exclusion criterion ensured that the $\mathrm{RB}$ effects were not biased by participants responding repeat as their default decision when they were unsure of their response. The final sample of 26 participants ( 21 females, five males) who had their data included in the analysis were aged between 17 and 65 years $\left(M_{\text {age }}=\right.$ $24.46, S D=11.60)$.

\section{Materials and RSVP Task Stimuli}

\section{POSITIVE AND NEGATIVE AFFECT SCHEDULE}

Affect has been shown to influence attentional resources (Flaisch, Junghöfer, Bradley, Schupp, \& Lang, 2007). Therefore, participants completed the Positive and Negative Affect Schedule (PANAS; Watson, Clark, \& Tellegan, 1988) to ensure that their mood state was within the normal range at the start of the experiment. Participants rated their current feelings (e.g., interested, distressed) on a five-point scale ( $1=$ not at all, $5=$ extremely) for each of the 10 positive and 10 negative affect items. Higher scores indicated higher levels of positive and negative affect.

\section{FACE AND FILLER STIMULI FOR THE RAPID SERIAL VISUAL PRESENTATION TASK}

Faces were taken from the NimStim facial set (Tottenham \& Nelson, 2000; Tottenham et al., 2009). The hair, ears, and clothing were removed from the photos of the faces to ensure identification could not occur through a single distinguishing feature (Allison, 2010). Thus, participants could only use the face to make their decisions about the gender, expression, and identity of the faces. Black and white photographs $\left(2.8^{\circ} \times 3.4^{\circ}\right.$ of visual angle) of 12 different individuals (six male, six female) were displayed on white backgrounds. Each face was displayed with a happy and an angry expression (24 targets in total). Angry and happy expressions were used, as they are regarded as being comparable (opposites) for identification purposes (Bruce \& Young, 1986; Ekman, 1999). Twelve filler stimuli were generated for use in the RSVP task. The filler stimuli were grayscale ovals placed randomly within a larger grey oval that were presented at the centre of a $4.8^{\circ} \times$ $4.8^{\circ}$ of visual angle white rectangular background. These filler items served as masks within each trial of the experiment.

\section{Procedure}

The experiment was run using the DMDX software (Forster \& Forster, 2003) on a Pentium IV 2.66GHz PC with a CRT flat screen monitor $(1,024 \times 724$ resolution). Participants were seated $60 \mathrm{~cm}$ from the computer screen in a dimly lit room and completed three different RSVP tasks (face identification, gender, and expression judgment tasks) in a one hour session. For all tasks, each trial began with a central fixation cross displayed for $500 \mathrm{~ms}$, followed by three filler items, C1, another filler item, C2, and three more filler items. As a result, each RSVP stream (trial) presented a nine-item sequence to participants. Single target trials included eight fillers and the critical item was presented in either the $\mathrm{C} 1$ or $\mathrm{C} 2$ position. Presentation rates were $90 \mathrm{~ms}$ per criti$\mathrm{cal}$ and filler item with no interstimulus interval. Figure 1 presents an 


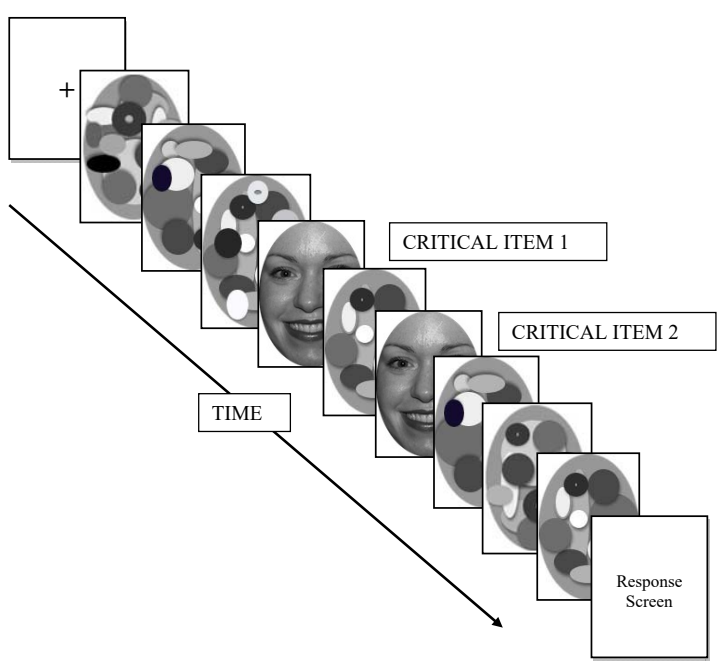

FIGURE 1.

Example of the sequence of stimuli presented on a complete repeat trial for the expression, gender and identity judgment tasks. Sample face is image 01 from Tottenham et al. (2009).

example trial sequence for a complete repeat trial for the expression, gender, and identity judgment tasks.

At the start of each of the three judgment tasks, participants were informed that either one face, two identical faces, or two different faces would be presented on each trial. Progression between trials was selfpaced (initiated by pressing the spacebar). There were four practice trials prior to each RSVP task, and task order was counterbalanced across participants to minimize practice effects. ${ }^{3}$

In each of the identity, gender, and expression judgment tasks, there were 96 trials, with each of the 12 happy and 12 angry faces being presented once in each of the four repetition conditions. The four repeat conditions included a complete repetition (same face and emotion), an emotion repetition (different face, same emotion), an identity repetition (same face, different emotion) and no repetition (different face, different emotion). An additional 10 trials, with only one critical item, were also included in each of the three RSVP tasks.

For the expression and identity judgment tasks, $\mathrm{C} 1$ and $\mathrm{C} 2$ were of the same gender to prevent decisions being made on gender differences alone, which would have negated the expression and identity judgments required by the task. As the same faces had to be used for the complete and identity repeat conditions, the same gender faces were also used for the emotion and no repeat trials to ensure consistency of $\mathrm{C} 1$ and $\mathrm{C} 2$ presentations across all four repeat conditions. Had faces of different genders been used for the emotion and no repeat trials, this would have highlighted the presentation of the two different faces and confounded the RB effects. In the gender judgments, if the same gender faces were shown on every trial, then every response for the task, except the single trials, would have been female-female or male-male, which would have undermined the true gender decisions required in this task. To overcome this issue, the same gender faces were shown on the complete and identity repeat trials and different gender faces were presented on the emotion and no repeat trials.

For the expression and gender judgments, participants wrote down the facial emotional expression/expressions (happy, angry) of the faces and face's gender/genders (male, female) they observed within each of the RSVP streams (one, two repeats, or two different expressions or genders). For the identity judgment task, two different faces (same gender) with happy and angry emotional expressions appeared at the end of each trial (four faces shown, numbered one to four). Participants wrote down the number of the face/faces (single face, two repeats of the same face, or two different faces) they had observed within the stream. Responses were noted in an answer sheet for each RSVP task.

\section{Design}

This study used a fully repeated-measures design. The independent variables were: Judgment Task (expression, identity, gender judgments) and Repeat Condition (complete, emotion, identity, no repeat). The dependent variables were the percentage of trials with $\mathrm{C} 1$ and $\mathrm{C} 2$ correct ${ }^{4}$ and the percentage correct for the single critical item trials. ${ }^{5}$

\section{RESULTS}

\section{Reliability Analysis of the Positive and Negative Affect Schedule}

The positive (Cronbach's $\alpha=.83$ ) and the negative (Cronbach's $\alpha=.81$ ) affect scales of the PANAS (Watson et al., 1988) were reliable (overall $=.94$ ). All participants had scores on these measures that fell within the population norm range (Crawford \& Henry, 2004; $M=27.26, S D$ $=6.16$ for positive affect; and $M=13.43, S D=3.67$ for negative affect). Thus, no participant's current positive or negative mood would have affected their task performance.

\section{Single Target Catch Trial Data}

A repeated-measures analysis of variance (ANOVA) was used to compare the accuracy data for the single target trials across the three judgment tasks. See Table 1 for relevant descriptive statistics. The effect of judgment task, $F(2,50)=6.97, p=.002, \eta_{\mathrm{p}}{ }^{2}=.22$, revealed poorer single target accuracy for the identity judgments than the expression, $t(25)=2.86, p=.008, d=0.79$, and gender judgments, $t(25)=3.16, p$ $=.004, d=0.88$. There was no difference in accuracy for the expression and gender judgments, $t(25)=0.00, p=1.00$. Therefore, the task difficulty was greater for the identification judgments than for the gender and expression judgments, which would be due to the use of unfamiliar faces. ${ }^{6}$ The impact of greater task difficulty would be to reduce the magnitude of RB for the identity judgments. Therefore, this difference in single target trial accuracy does not negate any RB effects observed in the identity judgments task data in the following analysis. 


\begin{tabular}{|c|c|c|c|c|c|c|c|c|}
\hline \multicolumn{9}{|c|}{ Repeat Condition } \\
\hline & $\begin{array}{c}\text { Complete } \\
\text { Repeat }\end{array}$ & $\begin{array}{c}\mathrm{RB} \\
\text { Complete } \\
\text { Repeat }\end{array}$ & $\begin{array}{c}\text { Emotion } \\
\text { Repeat }\end{array}$ & $\begin{array}{c}\mathrm{RB} \\
\text { Emotion } \\
\text { Repeat }\end{array}$ & $\begin{array}{l}\text { Identity } \\
\text { Repeat }\end{array}$ & $\begin{array}{c}\mathrm{RB} \\
\text { Identity } \\
\text { Repeat }\end{array}$ & No Repeat & $\begin{array}{c}\text { Single } \\
\text { Target Trials }\end{array}$ \\
\hline Judgment & $M(S E)$ & $M(S E)$ & $M(S E)$ & $M(S E)$ & $M(S E)$ & $M(S E)$ & $M(S E)$ & $M(S E)$ \\
\hline Expression & $26.92(6.20)$ & $59.46(6.23)$ & $55.61(5.74)$ & $30.77(5.18)$ & $83.49(3.30)$ & $2.89(1.43)$ & $86.38(2.63)$ & $97.31(1.31)$ \\
\hline Gender & $32.69(7.65)$ & $55.77(7.95)$ & $80.61(3.28)$ & $7.85(1.91)$ & $64.26(5.83)$ & $24.20(5.40)$ & $88.46(2.64)$ & $97.31(1.05)$ \\
\hline Identity & $28.69(7.60)$ & $26.66(8.14)$ & $50.16(5.33)$ & $5.13(3.88)$ & $47.28(4.56)$ & $8.01(4.14)$ & $55.29(4.33)$ & $90.77(2.14)$ \\
\hline
\end{tabular}

Note. RB Complete Repeat is difference between accuracy in no repeat and complete repeat conditions. RB Emotion Repeat is the difference between accuracy in the no repeat and emotion repeat conditions. RB Identity Repeat is the difference between accuracy in the no repeat and the identity repeat conditions.

\section{Repetition Blindness for the Face Expression, Gender, and Identity Judgment Tasks}

A $3 \times 4$ (Judgment Task [identity, gender, expression judgments], $\times$ Face Characteristic Repeat Condition [complete, emotion, identity, no repeat]), repeated-measures ANOVA was used to examine RB in the three face judgment tasks. The effects of face characteristic repeat condition, $F(3,75)=39.26, p<.001, \eta_{\mathrm{p}}{ }^{2}=.61$, and judgment task, $F(2,50)$ $=36.88, p<.001, \eta_{\mathrm{p}}{ }^{2}=.60$, and the interaction between judgment task and face characteristic repeat condition, $F(6,150)=14.82, p<.001, \eta_{\mathrm{p}}{ }^{2}$ $=.37$, were all significant. See Table 1 for relevant descriptive statistics. A series of specific planned comparisons (2-tailed) were used as follows to further examine this interaction effect. First, the occurrence of an $\mathrm{RB}$ effect for the complete repeat, emotion repeat, and identity repeat conditions was examined by comparing accuracy for each of these different repeat types to accuracy in the no repeat condition. This analysis was conducted separately for each of the judgment tasks. The second set of analyses compared the magnitude of the three different $\mathrm{RB}$ effects (complete, emotion, identity) to determine which type of repetition produced the largest $\mathrm{RB}$ effect within each judgment task. The third set of analyses compared the magnitude of the RB effect for each repeat type (complete, emotion, identity) across the three different judgment tasks to determine which decision produced the largest $\mathrm{RB}$ effect for the different repeat types.

\section{REPETITION BLINDNESS FOR THE COMPLETE, EMOTION, AND IDENTITY REPETITIONS}

There were large complete RB effects (reduced accuracy for the complete repeat compared to the no repeat condition) for the expression, $t(25)=9.55, p<.001, d=1.87$, gender, $t(25)=7.02, p<.001, d=$ 1.37 , and identity judgments, $t(25)=3.27, p<.001, d=0.64$.

Accuracy for the emotion repeat condition (same emotion, different face) was compared to accuracy in the no repeat condition to examine emotion RB. There was emotion RB for the expression, $t(25)$ $=5.94, p<.001, d=1.16$, and gender judgments, $t(25)=4.11, p<.001$, $d=0.81$, but not for the identity judgments, $t(25)=1.32, p=.198, d$ $=0.26$.
RB for repeated face identity was examined by comparing performance in the identity repeat condition (same face, different emotion) to performance in the no repeat condition. There was identity RB for the gender judgments, $t(25)=4.42, p<.001, d=0.87$, and the identity RB effect approached significance for the expression, $t(25)=2.00, p=.056$, $d=0.39$, and the identity judgments, $t(25)=1.94, p=.064, d=0.38$.

Thus, there was complete RB and identity RB effects for all three judgment tasks and emotion RB occurred for the expression and gender judgments.

\section{COMPARISON OF COMPLETE, EMOTION, AND IDENTITY REPETITION BLINDNESS EFFECTS WITHIN EACH JUDG- MENT TASK}

For the expression judgments, the complete RB effect was larger than the emotion RB effect, $t(25)=5.26, p<.001, d=1.03$, or the identity RB effect, $t(25)=9.43, p<.001, d=1.85$. The emotion RB effect was larger than the identity RB effect, $t(25)=5.80, p<.001, d=1.14$.

For the gender judgments, the complete RB effect was larger than the emotion $\mathrm{RB}, t(25)=6.21, p<.001, d=1.22$, and the identity $\mathrm{RB}$ effects, $t(25)=4.30, p<.001, d=0.84$. The identity RB effect was larger than the emotion RB effect, $t(25)=-2.82, p=.009, d=0.55$.

For the identity judgments, there was more complete RB than identity RB, $t(25)=2.80, p=.010, d=0.55$, and, as noted above, no emotion RB.

Thus, for the expression judgments, the complete RB effect was larger than the emotion RB effect, which was larger than the identity $\mathrm{RB}$ effect. In the gender judgment task, the complete RB effect was larger than the identity RB effect and smallest for the emotion RB effect. For the identity judgments, the complete RB effect was larger than the identity RB effect.

\section{COMPARISON OF COMPLETE, EMOTION, AND IDENTITY REPETITION BLINDNESS EFFECTS ACROSS THE JUDG- MENT TASKS}

The magnitude of the complete RB effect was larger in the expression, $t(25)=5.30, p<.001, d=1.04$, and gender judgments than the identity judgments, $t(25)=6.48, p<.001, d=1.27$. There was no differ- 
ence in the size of the complete RB effect for the expression and gender judgment tasks, $t(25)=0.75, p=.460, d=0.15$.

In comparing the magnitude of the emotion RB effect across the three tasks, the expression judgments showed a larger emotion RB effect than the gender judgments, $t(25)=4.07, p=.001, d=0.77$. As noted above, there was no emotion RB for the identity judgments.

The magnitude of the identity RB effect was larger in the gender than the expression judgments, $t(25)=3.95, p=.001, d=0.78$, and larger in the gender than the identity judgments, $t(25)=2.52, p=.018$, $d=0.50$. The magnitude of the identity RB effect did not differ for the expression and identity judgments, $t(25)=1.37, p=.183, d=0.27$.

Hence, the complete RB effect was larger for the expression and gender judgment tasks than the identity judgments. Emotion repetition produced more RB for the expression decisions than the gender judgments and the identity RB effect was larger for the gender than the identity and expression judgments.

\section{DISCUSSION}

This study compared RB for expression, gender, and identity judgments about faces to determine if the type of decision or the type of repeated face aspect affected the magnitude of the $\mathrm{RB}$ effects. There was a complete RB effect for all three judgment tasks. This is consistent with previous research for expression (complete repeat; Buttle, 2010), gender judgments of faces (Buttle, 2010), and unfamiliar and famous face identification (Coltheart, et al., 2004; Mondy, et al., 2004). Identity repeats (same face, different emotion) also produced RB in the three judgment tasks. This is consistent with previous studies showing identity RB for expression judgments (Buttle, 2010; Mowszowski et al., 2012), gender judgments (Buttle, 2010), and for famous faces with various expressions in a face identification judgment (Coltheart et al., 2004).

Across the three judgment tasks, the magnitude of the complete $\mathrm{RB}$ effect was larger than the identity RB effects and larger than the emotion $\mathrm{RB}$ effects for the expression and gender decisions. The larger complete $\mathrm{RB}$ effect relative to the identity $\mathrm{RB}$ effect for the expression and gender decisions is consistent with Buttle (2010) and could occur via repetition of all facial elements in the complete repeat condition and only identity repetition (not emotion) for the identity RB effects.

This study found emotion RB for the gender and expression judgments indicating that $\mathrm{RB}$ is not solely due to repeated face identity information but that it also occurs with repeated facial feature information (e.g., emotion). The emotion RB for the gender and expression judgments observed in this study contrasts with the lack of emotion RB reported by Buttle (2010) for these same decisions. The different results across the two studies may be due to a range of design differences. For example, this study used more faces than Buttle (current study used 12 faces, Buttle's, 2010, study-four faces) and all distinguishing features (e.g., hair) were removed from the face stimuli. These methodological modifications would have made the expression and gender judgments more difficult than in Buttle's study, thereby producing larger emotion $\mathrm{RB}$ effects. This idea is further supported by the fact that the complete repeat $\mathrm{RB}$ effects reported in this study for the gender and expression judgments were larger than those reported by Buttle (average of 26\% compared to the current study average of 57.25\%). The happy and sad faces used by Buttle were drawn from a different database than the happy and angry faces used in this study. Hence, the use of different emotions and faces that may have expressed different levels of emotionality may also account for the different emotion RB results across the two studies. Further, Buttle manipulated task as a betweensubjects factor, which was a within-subject variable in this study. Thus, any of these design differences may have contributed to the different outcomes observed here compared to Buttle.

The size of the complete RB effect was larger for the expression and gender judgments than face identity judgments. Accuracy for complete repeat trials was similar across all three judgment tasks, and therefore, this difference in RB magnitude was due to lower accuracy in the no repeat condition for the identity judgments compared to the other two judgment tasks. This outcome is consistent with the poorer overall single target accuracy for the identity than expression or gender judgments, confirming that the identity task was the most difficult decision. Importantly, the accuracy for the face identification task was comparable with previous RB studies (e.g., accuracy of 56 to $60 \%$ for the no repeat trials and 30 to $43 \%$ for the repeat trials; Coltheart et al., 2005; Harris \& Dux, 2005; Hayward et al., 2010). This indicates that the complete RB effect for the identity judgments is consistent with the RB effects observed in previous studies. These judgement task performance differences are also consistent with studies that have demonstrated slower processing speeds for making face identity decisions compared to gender decisions (Bruyer et al., 1993; Palermo \& Rhodes, 2007) and reports that expression judgments are so rapid that they occur almost without conscious awareness of the observer (Esteves, Dimberg, \& Öhman, 1994; Esteves, Parra, Dimberg, \& Öhman 1994; Murphy, Monohan, \& Zajonc, 1995; Murphy \& Zajonc, 1993).

There was more emotion $\mathrm{RB}$ for the expression than the gender judgments and no emotion RB for the identity judgments. While emotion RB for the expression judgments would be expected, as this was the relevant facial characteristic for the judgment, the emotion $\mathrm{RB}$ for the gender judgments is a new finding. Moreover, it indicates that within an RSVP task, the processing of emotional information is automatic (Batty \& Taylor, 2003; Mogg \& Bradley, 1999a, 1999b) when making decisions about gender. The lack of emotion RB for face identity judgments is consistent with models of face recognition that posit the independent processing of emotion and identity information (e.g., Bruce \& Young, 1986; Duchaine \& Yovel, 2015; Haxby et al., 2000). It is also consistent with neural evidence showing that the amygdala only responds to emotional expressions when there are sufficient attentional resources to process this aspect of the faces (Pessoa et al., 2002).

For example, within the expression judgment task, the face emotion was central to the task, and therefore, participants would have allocated their attentional resources to this feature during face processing. ${ }^{7}$ Further, as the gender and expression judgments were easier than the identity judgments, this allowed the participants to have additional attentional resources to process the face emotion during these tasks. 
Hence, for both the expression and gender judgments, the emotion of the face was attended to and tokenized upon presentation of Face 1. Therefore, when the emotion was repeated with Face 2, it could not be token individuated, and emotion RB occurred. In contrast, for the identity judgments, emotion was not encoded for either face as it was not relevant to the task decision, and the greater task difficulty limited the attentional capacity to process this task-irrelevant emotional information (Pessoa et al., 2002). This idea is supported by the similar performance accuracy in the emotion repeat and no repeat conditions for the identity judgment task, and it is consistent with Buttle's (2010) suggestion that for RB to occur participants need to encode the repeated level of information. The lack of emotion RB for the identity judgments provides behavioral data that supports Duchaine and Yovel's (2015) suggestion that the role of the fusiform face area in expression processing reflects a general sensitivity to shape information, and it is not an additional area for processing facial expression, as some have suggested (e.g., Bernstein \& Yovel, 2015).

Buttle (2010) reported that identity RB effects were of the same magnitude for gender and expression judgments. However, in this study, the identity RB effects were larger for the gender than the identity judgments and very small for the expression judgments. These results are consistent with participants attending to and encoding the overall face structure to make the gender and identity judgments. In contrast, when making expression judgments, participants focused their attention on this facial feature and ignored the overall face representation. This reduced identity RB, as the only face feature encoded was the nonrepeated emotion on the expression judgment trials, and thus, repeated face identity did not impact on task performance.

For the expression judgments, the size of the RB effect was larger for complete than emotion repeats, which was larger than for identity repeats. In contrast, for the gender judgments, the complete RB effect was larger than the identity RB effect, and the emotion RB effect was the smallest. The identity judgments showed a larger complete RB effect than the identity RB effect and no emotion RB. These results indicate that the repeated facial component is processed differently if that aspect is relevant to the attentional requirements of the judgment task.

According to the token individuation hypothesis, each judgment task produced RB for complete repeat trials because, although both $\mathrm{C} 1$ and C2 were typed, only C1 was token individuated (Kanwisher, 1987). The smaller identity than complete RB effects across the three tasks indicate that changing one feature (emotion) seems to assist with token individuation of the identity of the second face, thereby reducing identity RB. Emotion RB for the gender and expression judgments but not the identity judgments indicates that repeated presentation of one facial feature is sufficient to produce $\mathrm{RB}$ (only allow $\mathrm{C} 1$ to be token individuated), but the feature must be directly relevant to the task decision (operational during stimulus encoding). This idea is further supported by the larger emotion than identity RB effect in the expression judgment task, the emotion RB effect being larger for the expression than gender decisions and the identity RB effect being larger for the gender decisions than the identity and expression judgments.
The complete, identity, and emotion RB effects can also be explained in relation to seeing faces multiple times in quick succession during real world face-to-face interactions. For example, the large complete $\mathrm{RB}$ effects for all tasks suggest that when the same person with the same emotion is encountered, their face representation is not token individuated for a second time, as their unchanged representation is not informative during this time. The smaller identity than complete RB effects suggest that changes in emotion during interactions are sufficient to update the representation of that person on some occasions (token individuate the second face). This would be vital for assessing changes in their emotional state and updates on the progress of the social interaction. When assessing a person's identity, the lack of emotion RB (same emotion, different person) would provide the face recognition system with a strong signal that a new person has been encountered within the environment, thereby facilitating face recognition. In this case, emotion RB might slow down the face recognition processes which could have serious implications for survival. This contrasts with the encoding of facial emotion when making judgments about a person's expression and gender. As these more global facial assessments might be used in observing unfamiliar faces, it would be vital to encode their emotional state in the very initial stages of face encoding and only signal alterations in emotional states within a short time period. Hence, the complete, identity, and emotion RB effects for faces appear to play a functional role in the operation of our face recognition processes within real world environments.

In summary, the results of this study showed that the magnitude of the $\mathrm{RB}$ effect varied across the type of repeated facial information (e.g., more RB for complete than identity or emotion repeat conditions). It was also shown that the occurrence of complete, emotion, or identity RB effects for faces were affected by the task instructions, which directed participants to focus on certain aspects of the facial stimuli. Thus, the results of this study provide further support for the operation of multiple independent pathways (gender, expression, identity) within the face recognition system and suggest that task relevant facial characteristics and attentional resources can mediate the magnitude of $\mathrm{RB}$ for faces.

\section{FOOTNOTES}

${ }^{1}$ This explanation assumes that emotion RB does not occur, as everyday interactions with people require the continued updating of their emotional expressions to recognize the same person during these interactions. See Buttle (2010, p. 253) for the complete account.

${ }^{2}$ It should be noted that Buttle (2010) also conducted an analysis of the first quartile of task trials, and these data replicated the overall results. However, it could be suggested that 128 trials is sufficient to produce familiarity with four faces with hairstyles clearly visible, as it has been suggested learning new faces occurs quickly (Burton \& Jenkins, 2011).

${ }^{3}$ Task order was used as an independent variable in the initial analysis to ensure the counterbalancing had controlled for task order effects. There was no significant main effect or interaction between task order and judgment task or repeat condition $(F<2.14$, for all condi- 
tions, $p>.103$, for all conditions), confirming the counterbalanced task order did not affect the results. Therefore, task order was not included as an independent variable in the analysis reported within the results section.

${ }^{4}$ For the identity judgements, $\mathrm{C} 1$ and $\mathrm{C} 2$ were scored as correct if the right face was selected regardless of emotion, as this task required participants to identify the face or faces presented in the RSVP stream. This procedure also ensured that participants' response options were equivalent to the two response options that were used for the expression and gender judgement tasks. This scoring method also accounted for decisions about the face identity and not both the face identity and emotion.

${ }^{5}$ Accuracy data was not transformed to allow a direct comparison of the results of this study to those reported by Buttle (2010) who also used percentage correct as the dependent variable (DV). Other $\mathrm{RB}$ studies also typically report the percentage correct as the DV (e.g., Coltheart et al., 2005; Harris \& Dux, 2005; Hayward, Zhou, Man, \& Harris, 2010).

${ }^{6}$ An additional analysis using the data for only the correct faces and emotions for the identification task revealed the same outcomes as this analysis, with only marginally lower accuracy for these single trials. This supports the assumption that the poorer performance in the face identification task relative to the expression and gender judgments was due to the use of unfamiliar faces rather than the four response options being used in this task.

${ }^{7}$ It could be suggested that the emotion RB for the expression judgments was due to featural overlap (mouth curve) creating visual similarity and therefore, visual RB rather than emotion RB. However, this explanation cannot account for the emotion RB evident in the gender judgments, where participants would have attended to overall configural information. Thus, the evidence indicates the processing of facial emotion during expression and gender judgments in a RSVP task.

\section{AUTHOR'S NOTE}

We thank an anonymous reviewer for providing helpful feedback on an earlier version of this manuscript.

\section{REFERENCES}

Adolphs, R. (1999). The human amygdala and emotion. The Neuroscientist, 5, 125-137. doi: 10.1177/107385849900500216 Adolphs, R., Tranel, D., Damasio, H., \& Damasio, A. (1994). Impaired recognition of emotion in facial expressions following bilateral damage to the human amygdala. Nature, 372, 669-672. doi: 10.1038/372669a0 wWW

Allison, J. (2010). Emotions and their influence on visual attention: A study of repetition blindness for faces and facial expressions. Unpublished honors thesis, School of Applied Psychology, Griffith University, Gold Coast, Australia.

Andrews, T. J., Davies-Thompson, J., Kingstone, A., \& Young, A. W. (2010). Internal and external features of the face are represented holistically in face-selective regions of visual cortex. The Journal of Neuroscience, 30, 3544-3552. doi: 10.1523/ JNEUROSCI.4863-09.2010 $\overline{\mathrm{WWW}}$
Arnell, K., \& Jolicoeur, P. (1997). Repetition blindness for pseudoobject pictures. Journal of Experimental Psychology: Human Perception and Performance, 23, 999-1013. doi: 10.1037/00961523.23.4.999

Batty, M., \& Taylor, M. J. (2003). Early processing of the six basic facial emotional expressions. Cognitive Brain Research, 17,

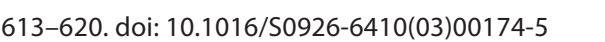

Bavelier, D. (1994). Repetition blindness between visually different items: The case of pictures and words. Cognition, 51,

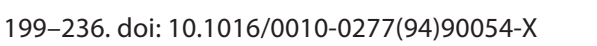

Bernstein, M., \& Yovel, G. (2015). Two neural pathways of face processing: A critical evaluation of current models. Neuroscience \& Biobehavioral Reviews, 55, 536-546. doi:

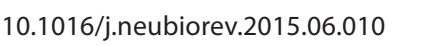

Bruce, V., \& Young, A. (1986). Understanding face recognition. British Journal of Psychology, 77, 305-327. doi: 10.1111/j.20448295.1986.tb02199.x WWW

Bruyer, R., Galvez, C., \& Prairial, C. (1993). Effect of disorientation on visual analysis, familiarity decision and semantic decision on faces. British Journal of Psychology, 84, 433-441. doi: 10.1111/j.2044-8295.1993.tb02494.x |WWW

Burton, A. M., \& Jenkins, R. (2011). Unfamiliar face perception. In A. Calder, G. Rhodes, M. Johnson, J. V. Haxby (Eds.), The Oxford handbook of face perception (pp. 287-306). Oxford, England: Oxford University Press

Buttle, H. (2010). Repetition blindness for faces reflects identity coding but not emotion coding. Perceptual and Motor Skills, 11, 245-256. doi: 10.2466/pms.110.1.245-256 WWW

Calder, A., Rowland, D., Young, A., Nimmo-Smith, I., Keane, J., \& Perrett, D. (2000). Caricaturing facial expressions. Cognition 75 , 1-43. doi: 10.1016/S0010-0277(00)00074-3 |wWW

Coltheart, V., Bornhofen, C., Mondy, S., \& Stephenson, L. (2004). Repetition blindness for famous faces [Abstract]. Perception, 33 (Suppl. 1), 14.

Coltheart, V., Mondy, S., \& Coltheart, M. (2005). Repetition blindness for novel objects. Visual Cognition, 12, 519-540. doi: $10.1080 / 13506280444000427$ WWW

Crawford, J. R., \& Henry, J. D. (2004). The Positive and Negative Affect Schedule (PANAS): Construct validity, measurement properties and normative data in a large nonclinical sample. British Journal of Clinical Psychology, 43, 245-265. doi: 10.1348/0144665031752934

Duchaine, B., \& Yovel, G. (2015). A revised neural framework for face processing. Annual Review of Vision Science, 1, 393-416.

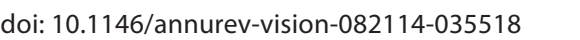

Ekman, P. (1999). Facial expressions. In T. Dalgleish \& M. Power (Eds.), Handbook of cognition and emotion (pp. 301-320). Chichester, England: John Wiley \& Sons Ltd.

Ellis, A., Flude, B., Young, A., \& Burton, A. (1996). Two loci of repetition priming in recognition of familiar faces. Journal of Experimental Psychology: Learning, Memory and Cognition, 22, 295-308. doi: 10.1037/0278-7393.22.2.295 WWW 
Ellis, A., Young, A., \& Flude, B. (1990). Repetition priming and face processing: Priming occurs within the system that responds to the identity of a face. Quarterly Journal Experimental Psychology, 42A, 495-512. doi: 10.1080/14640749008401234 |WWW|

Esteves, F., Dimberg, U., \& Öhman, A. (1994). Automatically elicited fear: Conditioned skin conductance response to masked facial expressions. Cognition and Emotion, 8, 393-413. doi: 10.1080/02699939408408949

Esteves, F., Parra, C., Dimberg, U., \& Öhman, A. (1994). Nonconscious associative learning: Pavlovian conditioning of skin conductance responses to masked fear-relevant facial stimuli. Psychophysiology, 31, 375-385. doi: 10.1111/j.14698986.1994.tb02446.x WWW

Fisher, K., Towler, J., \& Eimer, M. (2016). Facial identity and facial expression are initially integrated at visual perceptual stages of face processing. Neuropsychologia, 80, 115-125. doi: 10.1016/j.

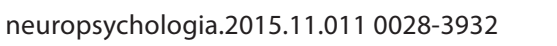

Flaisch, T., Junghöfer, M., Bradley, M., Schupp, H. T., \& Lang, P. (2007). Rapid picture processing: Affective primes and targets. Psychophysiology, 45, 1-10. doi: 10.1111/j.1469-8986 2007.00600.x WWW

Forster, K. I. (1970). Visual perception of rapidly presented word sequences of varying complexity. Attention, Perception, \& Psychophysics, 8, 215-221. doi: 10.3758/BF03210208

Forster, K. I., \& Forster, J. (2003). DMDX: A windows display program with millisecond accuracy. Behavior Research Methods, Instruments \& Computers, 35, 116-124. doi: 10.3758/ BF03195503 WWW

Gobbini, M. I., \& Haxby, J. V. (2007). Neural systems for recognition of familiar faces. Neuropsychologia, 45, 32-41. doi: 10.1016/j.

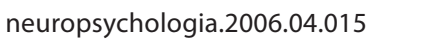

Habel, U., Windischberger, C., Derntl, B., Robinson, S., KryspinExner, I., Gur, R., \& Moser, E. . (2007). Amygdala activation and facial expressions: Explicit emotional discrimination versus implicit emotion processing. Neuropsychologia, 45, 2369-2377. doi: 10.1016/j.neuropsychologia.2007.01.023 WWW

Harris, I., \& Dux, P. (2005). Orientation-invariant object recognition: Evidence from repetition blindness. Cognition, 95, 73-93. doi: 10.1016/j.cognition.2004.02.006

Hasselmo, M., Rolls, E., \& Baylis, G. (1989). The role of expression and identity in the face-selective responses of neurons in the temporal visual cortex of the monkey. Behavioral Brain Research, 32, 203-218. doi: 10.1016/S0166-4328(89)80054-3 $\underline{W W W}$

Haxby, J., Hoffman, E., \& Gobbini, M. (2000). The distributed human neural system for face perception. Trends in Cognitive Sciences, 4, 223-233. doi: 10.1016/S1364-6613(00)01482-0 $\underline{W W W}$

Hayward, W., Zhou, G., Man, W., \& Harris, I. (2010). Repetition blindness for rotated objects. Journal of Experimental Psychology: Human Perception and Performance, 36, 57-73. doi: 10.1037/ $\mathrm{a} 0017447 \underline{\mathrm{Ww}}$
Hennenlotter, A., Schroeder, U., Erhard, P., Haslinger, B., Stahl, R., Weindl, A., . . . Ceballos-Baumann, A. O. (2004). Neural correlates associated with impaired disgust processing in pre-symptomatic Huntington's disease. Brain, 127, 1446. doi: 10.1093/brain/awh165 www

Hole, G., \& Bourne, V. (2010). Face processing. Oxford, England: Oxford University Press.

Holmes, A., Vuilleumier, P., \& Eimer, M. (2003). The processing of emotional facial expression is gated by spatial attention: Evidence from event-related brain potentials. Cognitive Brain Research, 16, 174-184. doi: 10.1016/S0926-6410(02)00268-9 WWW

Humphreys, G., Donnelly, N., \& Riddoch, M. (1993). Expression is computed separately from facial identity and it is computed separately for moving and static faces: Neuropsychological evidence. Neuropsychologia, 31, 173-181. doi: 10.1016/00283932(93)90045-2 $\overline{\text { WWW }}$

Jacobs, D., Shuren, J., Bowers, D., \& Heilman, K. (1995). Emotional face imagery, perception, and expression in Parkinson's disease. Neurology, 45, 1696-1702. doi: 10.1212/WNL.45.9.1696 WWW

Jehna, M., Neuper, C., Ischebeck, A., Loitfelder, M., Ropele, S., \& Langkammer, C., . . . Enziger, C. (2011). The functional correlates of face perception and recognition of emotional facial expressions as evidenced by fMRI. Brain Research, 1393, 73-83. doi: 10.1016/j.brainres.2011.04.007 |WWW

Kanwisher, N. (1987). Repetition blindness: Type recognition without token individuation. Cognition, 27, 117-143. doi: 10.1016/0010-0277(87)90016-3 WWW

Kanwisher, N., \& Yin, C (1993). Repetition blindness for pictures: Evidence for rapid computation of abstract visual descriptions. Investigative Ophthalmology and Visual Science, 34, 1131.

Kanwisher, N., Yin, C., \& Wojciulik, E. (1999). Phonological and visual codes in repetition blindness for words and pictures. In V. Coltheart (Ed.), Fleeting Memories (pp. 119-150). London, England: MIT Press/Bradford Books Series in Cognitive Psychology. $\underline{\underline{W W}}$

Mattson, A., Levin, H., \& Grafman, J. (2000). A case of prosopagnosia following moderate closed head injury with left hemisphere focal lesion. Cortex, 36, 125-137. doi: 10.1016/S00109452(08)70841-4 WwW

Mogg, K., \& Bradley, B. P. (1999a). Orienting of attention to threatening facial expressions presented under conditions of restricted awareness. Cognition \& Emotion, 13, 713-740. doi: 10.1080/026999399379050

Mogg, K., \& Bradley, B. P. (1999b). Some methodological issues in assessing attentional biases for threatening faces in anxiety: A replication study using a modified version of the probe detection task. Behavior Research and Therapy, 37, 595-604. doi:

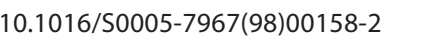

Mondy, S., Coltheart, V., \& Stephenson, L. (2004). Repetition effects on memory for unfamiliar faces under rapid serial visual 
presentation conditions [Abstract]. Perception, 33 (Suppl. 1), 109.

Mouchetant-Rostaing, Y., Giard, M., Bentin, S., Aguera, P., \& Pernier, J. (2000). Neurolo-physiological correlates of face sex processing in humans. European Journal of Neuroscience, 12, 303-310. doi: 10.1046/j.1460-9568.2000.00888.x

Mowszowski, L., McDonald, S., Wang, D., \& Bornhofen, C. (2012). Preferential processing of threatening facial expressions using the repetition blindness paradigm. Cognition and Emotion, 26, 1238-1255. doi: 10.1080/02699931.2011.648173 [WWW

Murphy, S., Monohan, J., \& Zajonc, R. (1995). Additivity and nonconscious affect: Combined effects of priming and exposure. Journal of Personality and Social Psychology, 69, 589-602. doi:

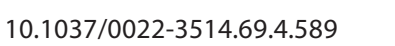

Murphy, S., \& Zajonc, R. (1993). Affect, cognition and awareness: Affective priming with optimal and suboptimal stimulus exposures. Journal of Personality and Social Psychology, 64, 723-739. doi: 10.1037/0022-3514.64.5.723 |wWW

Narumoto, J., Yamada, H., lidaka, T., Sadato, N., Fukui, K., Itoh, H., $\&$ Yonekura, Y. (2000). Brain regions involved in verbal or nonverbal aspects of facial emotion recognition. Neuroreport, 11, 2571-2576. doi: 10.1097/00001756-200008030-00044 |WWW

Palermo, R., \& Rhodes, G. (2007). Are you always on my mind? A review of how face perception and attention interact. Neuropsychologia, 45, 75-92. doi: 10.1016/j.

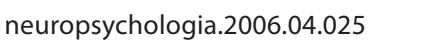

Pessoa, L., McKenna, M., Gutierrez, E., \& Ungerleider, L. G. (2002). Neural processing of emotional faces requires attention. Proceedings of the National Academy of Sciences of the United States of America, 99, 11458-11463. doi: 10.1073_ pnas. $172403899 \underline{\mathrm{WWW}}$

Potter, M. C., \& Levy, E. I. (1969). Recognition memory for a rapid sequence of pictures. Journal of Experimental Psychology, 81, 10-15. doi: 10. 10.1037/h0027470 Www

Sergent, J., Ohta, S., \& MacDonald, B. (1992). Functional neuroanatomy of face and object processing: A positron emis- sion tomography study. Brain, 115, 15-36. doi: 10.1093/ brain/115.1.15

Sprengelmeyer, R., Young, A., Calder, A., Karnat, A., Lange, H., Hömberg, V., ... Rowland, D. (1996). Loss of disgust: Perception of faces and emotions in Huntington's disease. Brain, 119, 1647-1665. doi: 10.1093/brain/119.5.1647 WWW

Todorov, A., \& Engell, A. (2008). The role of the amygdala in the implicit evaluation of emotionally neutral faces. Social Cognitive and Affective Neurosciences, 3, 303-312. doi: 10.1093/ scan/nsn033 WWW

Tottenham, N., \& Nelson, C. (2000). Research network on early experience and brain development: Face stimuli-emotion expression. Retrieved from http://www.macbrain.org/faces/ index.htm. Accessed on 29th March 2010.

Tottenham, N., Tanaka, J., Leon, A. C., McCarry, T., Nurse, M., Hare, T. A., ... Nelson, C.A. (2009). The NimStim set of facial expressions: judgments from untrained research participants. Psychiatry Research, 168, 242-249. doi: 10.1016/j.psychres.2008.05.006 WWW

Tranel, D., Damasio, A. R., \& Damasio, H. (1988). Intact recognition of facial expression, gender, and age in patients with impaired recognition of face identity. Neurology, 38, 690-690. doi: 10.1212/WNL.38.5.690 $\overline{\mathrm{WWW}}$

Watson, D., Clark, L., \& Tellegan, A. (1988). Development and validation of brief measures of positive and negative affect: The PANAS scales. Journal of Personality and Social Psychology, 54, 1063-1070. doi: 10.1037/0022-3514.54.6.1063 WWW

Wild, H., Barrett, S., Spence, M., O'Toole, A., Cheng, Y., \& Brooke, J. (2000). Recognition and sex categorization of adults' and childrens' faces in the absence of sex stereotyped cues. Journal of Experimental Child Psychology, 77, 261-299. doi: 10.1006/ jecp.1999.2554

Zhao, M., \& Hayward, W. (2010). Holistic processing underlies gender judgments of faces. Attention, Perception, and Psychophysics, 72, 591-596. doi: 10.3758/APP.72.3.591 |WWW

RECEIVED 31.01.2017| ACCEPTED 13.06.2017 